\section{Diabetes melito, tiazolidinedionas e fraturas: uma história inacabada}

\author{
Diabetes mellitus, thiazolidinediones and fractures: an unfinished story
}

André Gonçalves da Silva', Marise Lazaretti-Castro'
1 Unidade de Doenças Osteometabólicas, Serviço de Endocrinologia, Universidade Federal de São Paulo, Escola Paulista de Medicina (Unifesp-EPM), São Paulo, SP, Brasil

\title{
SUMMARY
}

The association of diabetes mellitus with increased fracture risk is well established, and is observed in both diabetes type 1 and type 2, due to multiple causes. Evidence from rodents suggests that thiazolidinediones (TZD), by activation of PPAR-gamma, cause increased bone marrow adiposity, with decreased osteoblastogenesis resulting in bone loss. Initial studies in humans evidence higher fracture risk in the population using these drugs, in comparison with other oral antidiabetic medications. TZD are largely prescribed for the treatment of type 2 diabetes, therefore, better understanding of their mechanisms of action and impact on bone mass and fracture risk is necessary, in order to guide the management of these patients in regards to prophylaxis and adequate treatment. This article summarizes current knowledge about the relationship between diabetes, TZD and fracture risk as well as, based on current evidence, tries to suggest ways to guide the population using these medications. Arq Bras Endocrinol Metab. 2010;54(4):345-51

\section{Keywords}

Thiazolidinediones; fractures; PPAR-gamma

\section{INTRODUÇÃO}

$\mathrm{D}$ iabetes melito (DM) é uma doença multissistêmica com alta prevalência e grande morbimortalidade, especialmente por complicações cardiovasculares, retinianas, renais e neurológicas. Entretanto, quadros osteoarticulares também costumam estar associados ao diabetes, como a artropatia de Charcot, o pé diabético e a osteoporose $(1,2)$. Os pacientes com DM apresentam aumento no risco de fraturas, que se manifesta de maneira mais evidente no DM tipo 1; pacientes portadores de DM tipo 1 possuem massa óssea reduzida quando comparados com controles pareados para idade e sexo. Por outro lado, os estudos curiosamente demonstram que no DM tipo 2, apesar de o aumento no risco de fraturas ser evidente, a densidade mineral óssea tende a ser igual ou superior à dos respectivos controles não diabéticos, sugerindo que fatores inerentes à condição diabética estariam prejudicando a qualidade óssea (3). Somando-se a esses aspectos, os estudos clínicos publicados recentemente demonstraram um aumento no 
risco de fraturas associado ao uso de tiazolidinedionas (TZD) no DM tipo 2, o que trouxe um novo olhar para essa indesejada complicação $(3,4)$. Este artigo pretende atualizar o leitor sobre esse tema, assim como sugerir possíveis abordagens para esse novo problema.

\section{DIABETES MELITO, RISCO DE FRATURAS E MASSA ÓSSEA}

A relação entre densidade mineral óssea (DMO), fraturas e diabetes melito varia de acordo com o tipo de diabetes e seu tempo de evolução. Um estudo prospectivo com 32.089 pacientes na pós-menopausa (Iowa Women's Health Study) mostrou que mulheres com DM 2 apresentaram risco 1,7 vez maior de fraturas de quadril que mulheres não diabéticas. Já pacientes com DM tipo 1 apresentaram dados mais exuberantes, com risco 12,25 vezes aumentado de desenvolvimento de fraturas de quadril em relação a mulheres não diabéticas, de idade semelhante (5). A determinação de uma relação de causalidade entre DM e aumento do risco de fratura mostra-se complexa, já que sabidamente outros aspectos não relacionados à doença propriamente dita podem interferir sobre o metabolismo ósseo, como a doença renal crônica, e sobre o risco de quedas, como as limitações visuais e a hipotensão postural, por exemplo. Além disso, algumas síndromes comumente associadas com fenótipo de DM possuem complicadores que levam à perda óssea, como o excesso de glicocorticoides na síndrome de Cushing e como na falência gonadal encontrada nas síndromes poliglandulares autoimunes e na hemocromatose (3). A associação de baixa densidade mineral óssea e DM está bem caracterizada no tipo 1, entretanto existe controvérsia na literatura sobre esse parâmetro quando se trata do DM tipo 2 . Enquanto alguns trabalhos encontraram DMO reduzida, outros trabalhos demonstraram DMO inalterada ou mesmo aumentada quando comparada a controles não diabéticos $(6,7)$.

Uma metanálise que avaliou DMO e risco de fraturas em pacientes com DM 1 e DM 12 observou que, ao contrário dos diabéticos tipo 1 , os pacientes com DM 12 avaliados apresentaram maior DMO no quadril $(\mathrm{Z}$ score: $0,27 \pm 0,01)$ e coluna lombar (Z score: $0,41 \pm$ $0,01)$ em relação a controles não diabéticos $(8)$. Apesar da maior DMO, o estudo evidenciou maior risco de fraturas nesses pacientes, o que foi confirmado em outros estudos, como pelo estudo de Roterdã e pelo estudo WHI. Embora ambos os estudos também te- nham encontrado maior massa óssea nos diabéticos, no primeiro o risco de fraturas foi $69 \%$ maior e no segundo $29 \%$ maior do que nos não diabéticos.

Dados da literatura sugerem que um maior índice de massa corpórea (IMC), sabidamente associado ao diabetes tipo 2 e à síndrome metabólica, possui um efeito atenuador sobre a perda óssea (3). O peso corporal, assim como o IMC, possui forte correlação com a densidade mineral óssea de maneira geral. Várias são as teorias que justificariam essa alta correlação e algumas direcionam esse efeito para as funções endócrinas do tecido adiposo, não somente pela aromatização dos androgênios aumentando os níveis estrogênicos, mas também pela produção de citocinas como adiponectina, leptina e resistina, que teriam algum papel na modulação da DMO nesses casos (3). De fato, alguns estudos demonstram efeito benéfico da adiponectina sobre o metabolismo ósseo, enquanto a leptina apresenta dados conflitantes sobre seu real papel. Diferentemente do DM 1, que se caracteriza por insulinopenia secundária à destruição imunomediada das células betapancreáticas, o DM 2 caracteriza-se por resistência periférica à insulina, com graus variáveis de hiperinsulinemia. A associação com sobrepeso ou obesidade é aqui bem estabelecida e esse parece ser o principal fator relacionado à maior $\mathrm{DMO}$ nestes pacientes em relação a controles não diabéticos ou com DM 1 . Apesar desse fato, o risco de fraturas encontra-se ainda aumentado. Várias hipóteses buscam explicar tal fato, como interações de hiperglicemia crônica com o sistema PTH/vitamina D, ou glicosilação não enzimática de várias proteínas ósseas, como colágeno tipo I, o que poderia levar à maior fragilidade óssea $(3,8)$.

A maior incidência de fraturas em pacientes com complicações crônicas é bem estabelecida, por associarse à maior limitação funcional e à maior incidência de quedas. A presença de neuropatia periférica em pacientes de idade mais avançada esteve isoladamente associada a maior risco de fraturas, aparentemente por levar à limitação de deambulação, à menor proteção dos pés e ao maior risco de quedas (3). Estudos animais sugerem que a interrupção da inervação habitual para determinado segmento ósseo leva à redução regional da massa óssea independentemente de alterações de carga mecânica (12). Por outro lado, nos grandes estudos epidemiológicos realizados para avaliar fatores de risco para fraturas, o DM não aparece como um fator de risco importante. Maiores estudos prospectivos são necessários para definir o real papel de cada uma dessas variáveis. 
Por não haver recomendações específicas, baseadas em evidências, para diagnóstico e tratamento da osteoporose no DM, parece razoável abordar todos os possíveis fatores de risco para fraturas nesta população, com destaque para o adequado tratamento do DM e para a prevenção de quedas. As sugestões de conduta, com exceção de um melhor controle glicêmico, restringem-se às mesmas utilizadas para pacientes com osteoporose de maneira geral, como adequação da ingestão de cálcio e vitamina $\mathrm{D}$, incentivos à atividade física regular, além da indicação de tratamento medicamentoso específico, segundo os mesmos critérios estabelecidos para osteoporose primária.

A prevalência de osteoporose varia intensamente entre os vários estudos existentes em populações de pacientes com DM tipo 1, a depender da idade e do tempo de duração da doença. Em crianças, os poucos estudos disponíveis apresentam alguns resultados discrepantes, mas que sugerem potencial efeito deletério do DM 1 sobre a aquisição do pico de massa óssea nestes pacientes. Em pacientes com idade superior, parece também haver tendência à menor massa óssea, como em estudo holandês que observou baixa massa óssea (T-score inferior a - 1 ) em 67\% dos homens $(\mathrm{n}=21$ ) e $57 \%$ das mulheres com DM 1, em comparação com grupo controle, pareado por idade $(\mathrm{n}=33)(13)$. Outro estudo, que recentemente revisou a literatura disponível sobre DM e risco de fraturas, concluiu que há associação de DM 1 e fraturas de quadril $(R R=6,3$, IC 95\%: 2,6-15,1), sendo esta mais exuberante que no DM $12(\mathrm{RR}=1,7, \mathrm{IC} 95 \%$ 1,3-2,2) (2). A maioria dos trabalhos analisados observou que a presença de complicações micro e macrovasculares, em resposta ao mau controle glicêmico de longa data, prediz menor massa óssea em pacientes com DM, mais do que a duração da doença em si. A associação de fatores genéticos, como polimorfismo do receptor de vitamina $\mathrm{D}$, na população de diabéticos tipo 1 , parece ter apenas pequena influência sobre a DMO.

Também aqui os dados mencionados ainda carecem de confirmação em estudos prospectivos, randomizados, especialmente desenhados para avaliar densidade mineral óssea e risco de fraturas nessa população. Nessas circunstâncias, parece prudente considerar tais pacientes como de risco para perda de massa óssea e fraturas e implementar precocemente medidas que possam diminuir esse risco, como ingesta adequada de cálcio de vitamina $\mathrm{D}$, prevenção de quedas e tratamento adequado do diabetes, reduzindo a incidência de complicações crônicas.

\section{TIAZOLIDINEDIONAS, MASSA ÓSSEA E FRATURAS}

Tiazolidinedionas (TZD), popularmente conhecidas como glitazonas, são drogas amplamente utilizadas no tratamento do diabetes tipo 2 (DM tipo 2). Por meio da ação sobre PPAR-gama, são drogas que promovem maior sensibilidade à insulina no músculo periférico e redução da produção hepática de glicose, além de inibição da lipólise periférica em adipócitos, redução dos níveis de ácidos graxos livres e da adiposidade visceral, resultando em melhora de parâmetros glicêmicos e metabólicos nesses pacientes. São drogas indicadas no tratamento de pacientes com DM tipo 2, apresentando bons resultados na manutenção do controle glicêmico em longo prazo, em comparação com outras opções terapêuticas consagradas, como sulfonilureias e metformina (9). Tais resultados tornam essa classe de drogas uma das mais prescritas no tratamento do diabetes tipo 2 . Além do bem estabelecido benefício das TZD no tratamento do DM tipo 2, tais drogas vêm sendo também utilizadas em outras patologias em que prevalece a resistência insulínica, como síndrome dos ovários policísticos $(\mathrm{SOP})$ e intolerância à glicose (10). Nesta última condição, o uso das TZD poderia retardar, ou mesmo evitar, a progressão para diabetes (9).

Dados de segurança publicados recentemente trouxeram preocupação com uma possível associação entre o uso crônico de rosiglitazona e o aumento no risco de eventos cardiovasculares, recentemente descrita, o que gerou certa parcimônia no uso das TZD na prática clínica (9). Soma-se a esse fato a publicação recente de trabalhos evidenciando perda de massa óssea e aumento de fraturas em pacientes usuárias dessas medicações. Uma revisão cuidadosa da literatura demonstra que tal observação já havia sido evidenciada em estudos animais, embora dados com pacientes em uso de TZD, tendo como desfecho DMO e fraturas, ainda não estejam disponíveis (8). Serão abordadas aqui as evidências atuais sobre a associação de DM tipo 2, massa óssea e fraturas, bem como sobre os efeitos osteometabólicos atribuídos às TZD, além de propor recomendações clínicas baseadas nessas evidências.

O mRNA de PPAR-gama é expresso nas células estromais da medula óssea, osteoblastos e precursores de osteoclastos. Muitos estudos concordam que há, via PPAR-gama, promoção de adipogênese nas células mesenquimais pluripotentes à custa de osteoblastogênese (4). Tal achado se dá pela supressão de marcadores osteoblásticos precoces, como Cbfa-1/Runx2, à 
semelhança do que ocorre espontaneamente em idades mais avançadas (11). Ratos com haploinsuficiência do PPAR-gama caracterizam-se por maior número de células osteoblásticas, em detrimento das adipocíticas, em culturas de células, em relação a controles. A ação do PPAR-gama nos osteoclastos é menos clara, com estudos destacando efeito estimulador, enquanto outros trabalhos não evidenciam ação relevante $(8,12)$.

Os agonistas PPAR-gama podem exercer efeitos substanciais sobre o metabolismo ósseo também de forma indireta. Por ação nesses receptores em tecido adiposo, há regulação da produção de adipocinas que, como anteriormente mencionado, podem ter efeitos importantes sobre a massa óssea (13). As TZD têm ação direta sobre a leptina, no entanto, essa ação não é consensual. Há alguns relatos descrevendo aumento e outros trabalhos, redução dos seus níveis (8). A expressão de adiponectina é estimulada pelas TZD. A redução dos níveis de insulina (possível agente anabólico sobre osteoblastos) (14), bem como de IGF-1, pode estar relacionada ao efeito dessas drogas.

Vários estudos prévios observaram perda de massa óssea em ratos ovariectomizados ou com idade avançada quando tratados com TZD, o que não foi observado em animais jovens. Lazarenko e cols. (11), por meio de microtomografia, histomorfometria e culturas de medula óssea, observaram relação inversa entre densidade mineral óssea (DMO) e teor de adipócitos na medula óssea de ratos. Constataram que, em animais adultos e idosos, o uso de rosiglitazona levou à redução na espessura e no número de trabéculas. Análise histomorfométrica, no entanto, evidenciou redução na taxa de formação óssea em ratos jovens, com redução do número de osteoblastos. Nos animais idosos tratados com a rosiglitazona, por sua vez, observou-se aumento da população osteoclástica, sem efeito aparente sobre os osteoblastos. Os autores interpretaram tais dados como resultado de um desbalanço da relação ligante do RANK/osteoprotegerina, que privilegiaria a diferenciação e o aumento da atividade dos osteoclastos no grupo tratamento.

Outro fator implicado foi a ativação de PPAR-gama 2 nas células mesenquimais medulares. Estas células, em ratos jovens, retêm potencial para diferenciação em osteoblastos ou adipócitos, a depender da ativação dos fatores de transcrição Runx-2 (runt-related transcription factor 2) ou PPAR-gama 2, respectivamente. Já em animais com idade avançada, aparentemente não se observou esse fenômeno. Resultados semelhantes foram evidenciados por Rzonca e cols. (15), que relataram redução de $24 \%$ no volume trabecular ósseo em ratos normais tratados com rosiglitazona $(20 \mathrm{mg} / \mathrm{kg} / \mathrm{dia})$ por 7 dias, com evidência histomorfométrica de prejuízo na formação óssea. Também neste estudo observouse maior adiposidade intramedular nos ratos tratados, $\mathrm{o}$ que condiz com os dados in vitro sobre diferenciação adipocitária preferencial das células mesenquimais, em detrimento da linhagem osteoblástica.

Até o momento, há poucos estudos publicados com seres humanos avaliando o efeito das TZD sobre a densidade mineral óssea. Estudos em pacientes tratados com troglitazona sugeriram possível efeito deletério dessa droga sobre o turnover ósseo, mas não sobre DMO após um ano. A interpretação de tais dados, no entanto, é difícil, devido ao pequeno tamanho da amostra e da falta de um grupo controle (12) (8). Dados retrospectivos também apontam para risco aumentado de fraturas (16). O primeiro grande estudo a relatar maior risco de fraturas em diabéticas usuárias de rosiglitazona foi o ADOPT (9). Este estudo seguiu 4.360 pacientes (42\% do sexo feminino, com idade média de 57 anos) por um período de cerca de quatro anos e tinha como objetivo primário avaliar a durabilidade do controle glicêmico (tempo até constatação de falha terapêutica, indicada pela elevação da glicemia de jejum) da monoterapia com rosiglitazona, em comparação com metformina e gliburida. Após a análise dos relatos de eventos adversos, observaram-se fraturas em 9,3\% das mulheres com rosiglitazona, contra $5,1 \%$ no grupo com metformina e $3,5 \%$ com a sulfonilureia (RR 2,18, IC 95\% 1,52-3,13 para rosiglitazona versus os demais tratamentos). Ocorreram em especial fraturas de membros inferiores. Não se observou diferença em fraturas de quadril entre os grupos assim como também não se observou aumento de fraturas entre os homens do estudo.

Análise posterior dos dados, recentemente publicada, não evidenciou qualquer outra diferença entre os grupos com potencial efeito sobre a massa óssea ou risco de fraturas (17). Subsequentemente foi relatado, inclusive pelo fabricante, maior risco de fraturas em pacientes em uso de pioglitazona $(18,19)$. Usando dados do estudo Health, Aging, and Body Composition Study (Health ABC), Schwartz e cols. (20), ao analisarem 69 pacientes em uso de TZD (idade de 70 a 79 anos), observaram, nas mulheres, perda óssea adicional por ano de uso da medicação, em comparação com os pacientes sem uso de TZD, de - $0,67 \%$ (IC 95\%: $-1,03,-0,30 \%$ ) em corpo total, - $1,14 \%$ (IC 95\%: - 1,90 , $-0,37 \%$ ) em coluna lombar, e $-0,38 \%$ (IC $95 \%$ : $-0,93$, $-0,17 \%)$ em quadril. 
A perda de massa óssea pode já ser observada após curto tempo de tratamento, como evidenciado por Grey e cols. (4). Estes autores estudaram 50 mulheres saudáveis, menopausadas, randomizadas para uso de rosiglitazona $8 \mathrm{mg} /$ dia ou placebo, com o objetivo primário de avaliar a variação dos marcadores de formação óssea após 14 semanas de tratamento. Após quatro semanas, os autores notaram redução significativa de PINP (marcador de formação óssea) de $13 \%$, sem alteração concomitante do marcador de reabsorção dosado (CTX). Após 14 semanas de seguimento, observou-se perda de massa óssea de 1,9\% no grupo tratado versus $0,2 \%$ no placebo $(P=0,003)$. Em outro trabalho recente, Berberoglu e cols. (21) demonstraram redução dos marcadores de formação óssea (fosfata se alcalina) em mulheres na pós-menopausa após 12 semanas de tratamento com TZD.

Metanálise recentemente publicada (com total de 4.400 mulheres e 7001 homens) confirmou maior risco de fraturas em mulheres em uso de rosiglitazona e pioglitazona (OR 2,23, IC 95\% 1,65-3,01; p > 0,001), não observando esse efeito em homens (OR 1,00, IC 95\% 0,73-1,39; p = 0,98) (22) (Tabela 1).

Embora a maior parte dos estudos evidencie risco aumentado em mulheres na pós-menopausa, sugerindo um possível efeito protetor dos esteroides sexuais, alguns dados implicam risco também para populações masculinas. Analisando dados de DMO de 32 homens com
DM 2 em uso de rosiglitazona, foi observada por Yaturu e cols. (23), após um período médio de 16 meses, uma diferença de perda óssea anual de $-1,05 \%$ em fềmur total, $-1,02 \%$ em colo femural e $-1,24 \%$ em coluna, em relação a 128 pacientes pareados por idade, sem uso de TZD. Apesar de ter sido um estudo observacional, sem ajuste para potenciais confundidores (24), ele chama a atenção por fornecer evidência de perda óssea relacionada à rosiglitazona também no sexo masculino. Artigo recente, em que mulheres jovens com síndrome dos ovários policísticos, com níveis elevados de testosterona, estradiol e insulina (todos esses fatores teoricamente protetores contra perda óssea), apresentou, após 16 semanas de tratamento com pioglitazona, redução da DMO $(1,1 \%$ em coluna lombar, e 1,4\% em fêmur) em relação ao placebo. Em função de a pioglitazona aparentemente aumentar o teor de gordura intramedular, a análise da DMO poderia estar subestimada nessas pacientes; contudo, elas apresentaram, também, menores níveis de fosfatase alcalina, sugerindo menor atividade osteoblástica, embora os níveis de osteocalcina não estivessem afetados (10).

Os dados da literatura apontam, portanto, para uma indução de perda óssea pelo uso crônico de TZDs, mais evidente entre as mulheres, mas possivelmente também presente entre os homens $(17,22)$. Resultados em estudos experimentais e decorrentes das variações dos marcadores de remodelação óssea sugerem que os efeitos da TZDs no tecido ósseo concentram-se sobre a diferen-

Tabela 1. Estudos avaliando efeitos das TZD sobre a densidade mineral óssea

\begin{tabular}{|c|c|c|c|c|c|c|c|c|}
\hline \multirow{3}{*}{ Estudo } & \multirow{3}{*}{ Desenho } & \multirow{3}{*}{ População } & \multirow{3}{*}{ Grupos (n) } & \multirow{3}{*}{ Desfechos } & \multicolumn{4}{|c|}{ Alteração da DMO, por sítio (\%) } \\
\hline & & & & & \multicolumn{2}{|c|}{ DMO coluna } & \multicolumn{2}{|c|}{ DMO quadril } \\
\hline & & & & & TZD & Controles & TZD & Controles \\
\hline $\begin{array}{l}\text { Grey e cols. } \\
\text { (4) }\end{array}$ & $\begin{array}{l}\text { Duplo-cego, } \\
\text { randomizado, } \\
\text { placebo controlado, } \\
\text { com } 14 \text { semanas } \\
\text { de duração }\end{array}$ & $\begin{array}{l}50 \text { pacientes do } \\
\text { sexo feminino, } \\
\text { com idade média } \\
\text { de } 68 \text { anos }\end{array}$ & $\begin{array}{c}\text { Rosiglitazona (25) } \\
\text { vs. placebo (25) }\end{array}$ & $\begin{array}{l}\text { DM0 de coluna } \\
\text { lombar e quadril; } \\
\text { marcadores de } \\
\text { metabolismo } \\
\text { ósseo }\end{array}$ & $-1,9 \pm 2,0$ & $-0,2 \pm 1,6$ & $-1,2 \pm 2,0$ & $-0,2 \pm 2,1$ \\
\hline $\begin{array}{l}\text { Glintborg e } \\
\text { cols. (10) }\end{array}$ & $\begin{array}{l}\text { Duplo-cego, } \\
\text { randomizado, } \\
\text { placebo-controlado, } \\
\text { com } 16 \text { semanas }\end{array}$ & $\begin{array}{l}44 \text { mulheres na } \\
\text { pré-menopausa, } \\
\text { com SOP e idade } \\
\text { média de } 32 \text { anos }\end{array}$ & $\begin{array}{c}\text { Pioglitazona (32) } \\
\text { vs. placebo (14) }\end{array}$ & $\begin{array}{c}\text { DMO e } \\
\text { marcadores de } \\
\text { metabolismo } \\
\text { ósseo }\end{array}$ & $-0,4 \pm 2,03$ & $0,67 \pm 2,46$ & $-1,02 \pm 2,6$ & $0,34 \pm 2,78$ \\
\hline $\begin{array}{l}\text { Schwartz e } \\
\text { cols. }(20)\end{array}$ & $\begin{array}{c}\text { Estudo Health } \\
\text { ABC: estudo de } \\
\text { coorte prospectivo } \\
\text { (4 anos) }\end{array}$ & $\begin{array}{c}666 \text { pacientes } \\
\text { diabéticos, com e } \\
\text { sem uso prévio de } \\
\text { TZD, com idade de } \\
\text { 70-79 anos }\end{array}$ & $\begin{array}{l}\text { Troglitazona (22) } \\
\text { vs. pioglitazona } \\
\quad(30) \text { vs. } \\
\text { rosiglitazona (31) } \\
\text { vs. controles (597) }\end{array}$ & $\begin{array}{c}\text { Variação } \\
\text { percentual da } \\
\text { DMO (bienal) }\end{array}$ & $\begin{array}{r}\text { Mulh } \\
(\mathrm{IC}-1,04-0,0 \\
\text { Hom } \\
(\mathrm{IC}-0,61-0,2\end{array}$ & $\begin{array}{l}\text { \%: -0,4 } \\
\text { \%ariação/ano } \\
\text { \% variação/ano }\end{array}$ & $\begin{array}{r}\text { Mulhere } \\
-2,06-0,4) . \mathrm{Hc} \\
-1,1-0,6) \%\end{array}$ & $\begin{array}{l}-1,23 \text { (IC } \\
\text { ens: -0,25 (IC } \\
\text { ariação/ano }\end{array}$ \\
\hline $\begin{array}{l}\text { Yaturu e } \\
\text { cols. (23) }\end{array}$ & $\begin{array}{l}\text { Estudo } \\
\text { retrospectivo, com } \\
\text { seguimento médio } \\
\text { de } 16 \text { meses }\end{array}$ & $\begin{array}{l}160 \text { homens com } \\
\text { DM tipo 2, com } \\
\text { idade média de } 69 \\
\text { anos }\end{array}$ & $\begin{array}{l}\text { Rosiglitazona (32) } \\
\text { vs. controles (128) }\end{array}$ & $\begin{array}{c}\text { Variação } \\
\text { percentual da } \\
\text { DMO }\end{array}$ & $-1,19 \pm 1,8$ & $-0,14 \pm 1,9$ & $0,69 \pm 2,4$ & $2,3 \pm 2,9$ \\
\hline
\end{tabular}

DMO: densidade mineral óssea; SOP: síndrome do ovário policístico; TZD: tiazolidinedionas. Adaptada de Loke e cols. (22). 
ciação e a atividade dos osteoblastos, portanto sobre a formação óssea. Em teoria, tal efeito seria mais deletério em mulheres na perimenopausa, momento no qual a reabsorção óssea estaria "fisiologicamente" aumentada.

Os laboratórios que comercializam produtos dessa classe terapêutica enviaram alertas à comunidade médica, notificando o aumento da prevalência de fraturas com o uso de TZDs e sugerindo que esse risco seja levado em consideração no momento da decisão terapêutica $(19,25)$. O FDA manifestou-se também solicitando que tais achados e recomendações fossem incluídas nas bulas dos medicamentos $(26,27)$. Até o momento, a recomendação dos fabricantes das TZD disponíveis no mercado é para considerar o risco de fraturas em todos os pacientes em uso dessas drogas, em especial no sexo feminino, realizando a profilaxia e o tratamento da perda de massa óssea de acordo com as recomendações correntes.

Apesar desses cuidados, várias questões permanecem em aberto, aguardando que novos estudos identifiquem as causas, os grupos de maior risco e as medidas indicadas. Há dúvidas se o dano induzido pelas TZD pode ser revertido após sua suspensão e quais seriam os sítios de maior risco. Também não se sabe se essa perda óssea pode ser minimizada, ou mesmo prevenida, por outras intervenções, como bisfosfonatos, cálcio ou vitamina D.

$\mathrm{O}$ que fazer? Pacientes em uso dessas medicações devem ser orientadas a respeito desse possível efeito deletério. Deve-se frisar que não existe, até o momento, recomendação oficial para avaliação e/ou seguimento diferenciado de tais pacientes quanto à massa óssea, estejam em uso ou não dessas drogas. É possível que o uso de TZD deva ser incluído na lista de fatores de risco para osteoporose e que sua indicação deva ser reconsiderada em pacientes com outros fatores de risco para osteoporose e fraturas, mas ainda é preciso grandes estudos, desenhados para tal fim, antes de uma posição definitiva. É necessário ainda que as grandes agências reguladoras exijam da indústria farmacêutica a realização de trabalhos dessa natureza, a fim de fornecer à classe médica subsídios que permitam julgar, de forma mais completa, a indicação e o risco-benefício dessas drogas para os pacientes.

Declaração: os autores declaram não haver conflitos de interesse científico neste estudo.

\section{REFERÊNCIAS}

1. Schwartz AV. Diabetes mellitus: does it affect bone? Calcif Tissue Int. 2003;73(6):515-9.
2. Janghorbani M, Van Dam RM, Willett WC, Hu FB. Systematic review of type 1 and type 2 diabetes mellitus and risk of fracture. Am J Epidemiol. 2007;166(5):495-505.

3. Hofbauer LC, Brueck CC, Singh SK, Dobnig H. I. Osteoporosis in patients with diabetes mellitus. J Bone Miner Res. 2007;22(9):1317-28.

4. Grey A, Bolland M, Gamble G, Wattie D, Horne A, Davidson J, et al. The peroxisome proliferator-activated receptor-gamma agonist rosiglitazone decreases bone formation and bone mineral density in healthy postmenopausal women: a randomized, controlled trial. J Clin Endocrinol Metab. 2007;92(4):1305-10.

5. Nicodemus KK, Folsom AR. Type 1 and type 2 diabetes and incident hip fractures in postmenopausal women. Diabetes Care. 2001;24(7):1192-7.

6. Perez-Castrillon JL, De Luis D, Martín-Escudero JC, Asensio T, del Amo R, Izaola O. Non-insulin-dependent diabetes, bone mineral density, and cardiovascular risk factors. J Diabetes Complications. 2004;18(6):317-21.

7. Tuominen JT, Impivaara O, Puukka P, RönnemaaT. Bone mineral density in patients with type 1 and type 2 diabetes. Diabetes Care. 1999;22(7):1196-200.

8. Grey A. Skeletal consequences of thiazolidinedione therapy. Osteoporos Int. 2008;19(2):129-37.

9. Kahn SE, Haffner SM, Heise MA, Herman WH, Holman RR, Jones $\mathrm{NP}$, et al. Glycemic durability of rosiglitazone, metformin, or glyburide monotherapy. N Engl J Med. 2006;355(23):2427-43.

10. Glintborg D, Andersen M, Hagen $C$, Heickendorff L, Hermann AP. Association of pioglitazone treatment with decreased bone mineral density in obese premenopausal patients with polycystic ovary syndrome: a randomized, placebo-controlled trial. J Clin Endocrinol Metab. 2008;93(5):1696-701.

11. Lazarenko OP, Rzonca SO, Hogue WR, Swain FL, Suva LJ, LeckaCzernik B. Rosiglitazone induces decreases in bone mass and strength that are reminiscent of aged bone. Endocrinology. 2007;148(6):2669-80.

12. Grey AB. Skeletal toxicity of thiazolidinediones. Ann Intern Med. 2008;148(7):563.

13. Yki-Jarvinen H. Thiazolidinediones. N Engl J Med. 2004;351(11): 1106-18.

14. Cornish J, Callon KE, Reid IR. Insulin increases histomorphometric indices of bone formation In vivo. Calcif Tissue Int. 1996;59(6):492-5.

15. Rzonca SO, Suva LJ, Gaddy D, Montague DC, Lecka-Czernik B. Bone is a target for the antidiabetic compound rosiglitazone. Endocrinology. 2004;145(1):401-6.

16. Meier C, Kraenzlin ME, Bodmer M, Jick SS, Jick H, Meier CR. Use of thiazolidinediones and fracture risk. Arch Intern Med. 2008;168(8):820-5.

17. Kahn SE, Zinman B, Lachin JM, Haffner SM, Herman WH, Holman $\mathrm{RR}$, et al. Rosiglitazone-associated fractures in type 2 diabetes: an Analysis from A Diabetes Outcome Progression Trial (ADOPT). Diabetes Care. 2008;31(5):845-51.

18. Earnshaw SR, Graham CN, Ettinger B, Amonkar MM, Lynch NO, Middelhoven $\mathrm{H}$. Cost-effectiveness of bisphosphonate therapies for women with postmenopausal osteoporosis: implications of improved persistence with less frequently administered oral bisphosphonates. Curr Med Res Opin. 2007;23(10):2517-29.

19. Spanheimer R. Re: observation of an Increased Incidence of Fractures in Female Patients Who Received Long-Term Treatment with ACTOSO (pioglitazone HOI) Tablets for Type 2 Diabetes Mellitus. 2007 (citado em 15/06/2008]. Disponível em: http://www.fda.gov/ medwatch/safety/2007/Actosmar0807.pdf 
20. Schwartz AV, Sellmeyer DE, Vittinghoff E, Palermo L, Lecka-Czernik $B$, Feingold KR, et al. Thiazolidinedione use and bone loss in older diabetic adults. J Clin Endocrinol Metab. 2006;91(9):3349-54.

21. Berberoglu Z, Gursoy A, Bayraktar N, Yazici AC, BascilTutuncu N, Guvener Demirag N. Rosiglitazone decreases serum bone-specific alkaline phosphatase activity in postmenopausal diabetic women. J Clin Endocrinol Metab. 2007;92(9):3523-30.

22. Loke YK, Singh S, Furberg CD. Long-term use of thiazolidinediones and fractures in type 2 diabetes: a meta-analysis. CMAJ. 2009;180(1):32-9.

23. Yaturu S, Bryant B, Jain SK. Thiazolidinedione treatment decreases bone mineral density in type 2 diabetic men. Diabetes Care. 2007;30(6):1574-6.
24. Schwartz AV, Sellmeyer DE. Thiazolidinedione therapy gets complicated: is bone loss the price of improved insulin resistance? Diabetes Care. 2007;30(6):1670-1.

25. Cobitz A. Clinical Trial observation of an increased incidence of fractures in female patients who received long-term treatment with Avandia ${ }^{\circledR}$ (rosiglitazone maleate) Tablets for Type 2 diabetes mellitus. 2007. Disponível em: http://www.fda.gov/medwatch/safety/2007/Avandia_GSK_Ltr.pdf

26. Safety alerts for drugs, biologics, medical devices, and dietary supplements; 2007 (citado em: 9/3/2007]; FDA Alert. Disponível em: http://www.fda.gov/medwatch/safety/2007/safety07.htm\#actos

27. 2007 Medicare safety: security alerts 2007 Fev (citado em 20/2/2007]. Disponível em: http://www.fda.gov/medwatch/safety/2007/safety07.htm\#rosiglitazone 